\title{
Tail Transitions in Queues with Long Range Dependent Input
}

\author{
Tim Daniëls and Chris Blondia \\ University of Antwerp, Dept. Mathematics and Computer Science, \\ Universiteitsplein 1, B-2610 Antwerpen \\ blondia@uia.ua.ac.be, \\ http://win-www.uia.ac.be/u/pats
}

\begin{abstract}
This paper studies the tail of the buffer occupancy distribution of a queueing system with $c$ parallel deterministic servers, infinite buffer capacity and an input process with consists of a superposition of a long range dependent on-off source and a batch renewal process. In particular, we investigate the decay of the tail for different values of $c$ and for different compositions of the traffic mix. It is shown that for $c=1$ (i.e. a single server system), the tail has a power law decay, while for $c>1$, different cases may be distinguished: if the arrival rate of the background traffic is larger than $c-1$, then the tail probabilities decay according to a power law, while for the other case, the decay is exponential.
\end{abstract}

\section{Introduction}

In the past years, traffic measurements on packet networks (e.g. Ethernet LAN traffic [1], VBR video streams over ATM [8]) have shown that the autocorrelation function of the number of arrivals in a time interval does not decrease exponentially in time, but according to a power-law. This type of traffic is referred to as long range dependent traffic (LRD), in contrast with the more classical short range dependent traffic (SRD) (e.g. Markovian models). The autocorrelation structure of the arrival process has a major impact on the buffer occupancy of a queueing system with this process as input. For Markovian traffic, the tail of the queue length distribution decays exponentially, while for LRD input traffic the tail of the buffer distribution decays according to a power law (see e.g. 5, [7], [9], [10] ). In [2, an exact formula for the buffer asymptotics of a discretetime queue with an LRD M/G/ $\infty$ input process was obtained. The $M / G / \infty$ is defined as a process where trains arrive in a time slot according to a Poisson process with rate $\lambda$. Each train consists of a number of back-to-back arrivals with length $\tau_{A}$, with distribution given by $\mathbf{P}\left\{\tau_{A}=k\right\} \sim a k^{-s}$, with $2<s<3$ and $a>0$. The buffer asymptotics are then determined by

$$
\mathbf{P}\{q>k\} \sim \frac{\lambda a \rho^{s-2}}{(s-2)(s-1)(1-\rho)} k^{2-s},
$$

where $q$ denotes the buffer occupancy and $\rho$ is the load of the system.

In this paper we consider a queueing system where the input process consists

G. Pujolle et al. (Eds.): NETWORKING 2000, LNCS 1815, pp. 264274 2000.

(C) Springer-Verlag Berlin Heidelberg 2000 
of a mix of LRD and SRD traffic. The LRD component is an on-off source with geometrically distributed off-period and on-periods which have a Paretolike distribution. The SRD component consists of a batch renewal process. To model the difference in speed of the input and output lines, we assume that this traffic mix is fed to a multi-server queue with $c$ parallel servers. The aim of the paper is to investigate the tail of the buffer occupancy distribution of this $(\mathrm{LRD}+\mathrm{SRD}) / \mathrm{D} / \mathrm{c}$ queue for different compositions of the traffic mix and different values of $c$. We show that when $c=1$, the tail has a power law decay, while for $c>1$, different cases may be distinguished: if the arrival rate of the background traffic is larger than $c-1$, then the tail probabilities decay according to a power law, while for the other case, i.e. the arrival rate of the background traffic is less than $c-1$, the decay is exponential. With respect to the transition point (i.e. when the arrival rate of the background traffic is exactly $c-1$ ), no conclusions can be drawn.

These results give some insight about the influence of spacing cells of an LRD traffic stream on the queueing behavior. The number of servers $c$ is a measure for the spacing distance. The results show that by spacing an LRD traffic stream and mixing with SRD traffic, a tail which decays according to a power law may be changed into an exponential decay.

\section{The Queueing System}

The queueing system that is considered consists of a multiplexer with $c$ servers each having a deterministic service time of one time slot, an infinite capacity buffer and input traffic which is the superposition of an on-off source and socalled background traffic. The on-off source has geometrically distributed offperiods and on-periods which have a Pareto-like distribution. Let $\tau_{A}$ be the duration of the on-periods and let $a_{k}=\mathbf{P}\left\{\tau_{A}=k\right\} \sim a k^{-s}$, with $2<s<3$ and $a>0$. For these parameters the on-off source generates LRD traffic. Indeed, if $X_{k}$ denotes the number of arrivals generated by the on-off source in slot $k$, then the results presented in [15] imply that

$$
\operatorname{Var}\left(X_{1}+\ldots+X_{n}\right) \sim \frac{\mathbf{E}\left[\tau_{B}\right]^{2}}{\left(\mathbf{E}\left[\tau_{A}\right]+\mathbf{E}\left[\tau_{B}\right]\right)^{3}} \frac{a}{(4-s)(3-s)(s-1)} n^{4-s}
$$

Hence by [14, the source generates LRD traffic with Hurst parameter $H=$ $(4-s) / 2$. The generating function associated with the off-periods is given by

$$
\frac{(1-\beta) z}{1-\beta z}
$$

Let $A(z)=\sum_{k=1}^{\infty} a_{k} z^{k}$. The background traffic generates $X_{n}$ arrivals in slot $n$, with $\left\{X_{n}, n \geqslant 1\right\}$ a sequence of i.i.d. random variables. The corresponding generating function is denoted by $\phi(z)$.

In what follows, we describe the input traffic using a matrix-analytic notation. The LRD on-off source can be considered as a D-BMAP (see [1]) as follows. Let 
$p_{n}^{a}$ be the probability that the LRD source is in the $(n+1)^{t h}$ slot of an on-period given that it was in the $n^{\text {th }}$ slot of this on-period in the previous slot. Then

$$
p_{n}^{a}=\frac{1-\sum_{i=1}^{n} a_{i}}{1-\sum_{i=1}^{n-1} a_{i}}
$$

Letting the first state of the LRD source be the off-state, then the generating function defining the transitions of the corresponding D-BMAP, is given by

$$
\mathbf{D}(z)=\left(\begin{array}{cccccc}
\beta & (1-\beta) z & 0 & 0 & 0 & \ldots \\
1-p_{1}^{a} & 0 & p_{1}^{a} z & 0 & 0 & \ldots \\
1-p_{2}^{a} & 0 & 0 & p_{2}^{a} z & 0 & \ldots \\
1-p_{3}^{a} & 0 & 0 & 0 & p_{3}^{a} z & \ldots \\
\vdots & \vdots & \vdots & \vdots & \vdots & \ddots
\end{array}\right) .
$$

Remark that $\mathbf{D}(z)$ is an infinite matrix. By the special structure of $\mathbf{D}(z)$ it is possible to obtain an implicit equation for its Perron-Frobenius eigenvalue $\lambda(z)$ :

$$
\lambda(z)=\beta+(1-\beta) A\left(\frac{z}{\lambda(z)}\right) .
$$

The generating function of the D-BMAP describing the superposition of the onoff source and the background traffic is given by $\phi(z) \mathbf{D}(z)$ with mean arrival rate

$$
\rho=\phi^{\prime}(1)+\frac{(1-\beta) A^{\prime}(1)}{(1-\beta) A^{\prime}(1)+1} .
$$

\section{The Tail Probabilities}

Consider the $(\mathrm{LRD}+\mathrm{SRD}) / \mathrm{D} / \mathrm{c}$ queue defined in the previous section. We consider two cases, namely $c=1$ and $c>1$.

\subsection{The Tail Probabilities When $c=1$}

Since there is only one server, the queueing model is a simple D-BMAP/D/1 queue. Define the generating function of the buffer occupancy as

$$
Q(z) \stackrel{\text { def }}{=} \sum_{k=0}^{\infty} q_{k} z^{k}
$$

with $q_{k}$ the steady-state probability of having $k$ customers in this system. Clearly $Q(z)=\mathbf{X}(z) \mathbf{e}$, with $\mathbf{X}(z)$ given by

$$
\mathbf{X}(z)=(z-1) \mathbf{x}_{0} \phi(z) \mathbf{D}(z)(z \mathbf{I}-\phi(z) \mathbf{D}(z))^{-1} .
$$

This expression is the Pollachek-Kinchin equation for the D-BMAP/D/1 queue, with the input determined by the generating function $\phi(z) \mathbf{D}(z)$. Since there is 
only one off-state, the vector $\mathbf{x}_{0}$ is given by $\mathbf{x}_{0}=(1-\rho 00 \ldots)$. The structure of $\mathbf{D}(z)$ allows us to compute the vector

$$
\mathbf{w}(z) \stackrel{\text { def }}{=} \mathbf{x}_{0} \phi(z) \mathbf{D}(z)(z \mathbf{I}-\phi(z) \mathbf{D}(z))^{-1},
$$

with $\mathbf{w}(z)=\left(w_{0}(z) w_{1}(z) \ldots\right)$. Some straightforward algebra leads to

$$
\begin{aligned}
& w_{0}(z)=\frac{\beta \phi(z) z+(1-\beta) \phi(z) A(\phi(z)) z}{z^{2}-\beta z \phi(z)-A(\phi(z))(1-\beta) z \phi(z)}, \\
& w_{1}(z)=\frac{(1-\beta) z^{2}}{z^{2}-\beta z \phi(z)-A(\phi(z))(1-\beta) z \phi(z)},
\end{aligned}
$$

and for $k \geq 2$,

$$
w_{k}(z)=\left[1-\sum_{i=1}^{k-1} a_{i}\right] \phi(z)^{k} w_{1}(z) .
$$

Since $Q(z)=\mathbf{X}(z) \mathbf{e}=(1-\rho)(z-1) \mathbf{w}(z) \mathbf{e}$, it follows that

$$
Q(z)=(1-\rho)(z-1)\left[w_{0}(z)+w_{1}(z) \frac{1-A(\phi(z))}{1-\phi(z)}\right] .
$$

By calculating $Q^{\prime}(1)$ we obtain

$$
\mathbf{E}[q]=\sum_{k=0}^{\infty} \mathbf{P}\{q>k\}=\frac{A^{\prime \prime}(1)}{2} \frac{1}{\mathbf{E}\left[\tau_{A}\right]+\mathbf{E}\left[\tau_{B}\right]}\left(\frac{\phi^{\prime}(1)^{2}}{1-\rho}+\phi^{\prime}(1)\right)+\Omega,
$$

with $\Omega$ denoting a term involving the finite quantities $\beta, \phi^{\prime}(1), \phi^{\prime \prime}(1)$ and $A^{\prime}(1)$ (we suppose that all the moments of the background traffic exist). Since $\mathbf{E}\left[\tau_{A}^{2}\right]=$ $\infty, Q^{\prime}(z)$ diverges when $z$ approaches 1 .

The Tauberian Theorem for power series (see e.g. [6]) plays a central role in the derivation of an expression for the tail probabilities of the buffer occupancy.

Theorem 1 (Tauberian theorem for power series). Let $p_{k} \geqslant 0$ and suppose that

$$
P(z)=\sum_{k=0}^{\infty} p_{k} z^{k}
$$

converges for $0 \leqslant z<1$. If $L$ is a constant and $0 \leqslant \sigma<\infty$, then the following relations are equivalent:

$$
\begin{gathered}
P(z) \sim \frac{L}{(1-z)^{\sigma}} \text { for } z \rightarrow 1- \\
p_{0}+p_{1}+\ldots+p_{n-1} \sim \frac{L}{\Gamma(\sigma+1)} n^{\sigma} \text { for } n \rightarrow \infty .
\end{gathered}
$$


This theorem leads to an expression for the behaviour of $Q^{\prime}(z)$ near 1 .

Lemma 1. The behaviour of $Q^{\prime}(z)$ near 1 is given by

$$
Q^{\prime}(z) \sim \frac{L}{(1-z)^{3-s}} \text { for } z \rightarrow 1-
$$

with

$$
L=\frac{a \Gamma(3-s)}{s-1} \frac{1}{\mathbf{E}\left[\tau_{A}\right]+\mathbf{E}\left[\tau_{B}\right]}\left(\frac{\phi^{\prime}(1)^{s-1}}{1-\rho}+\phi^{\prime}(1)^{s-2}\right) .
$$

Proof. To prove the statement (7) we rewrite $Q(z)$ :

$$
Q(z)=(1-\rho)\left(f_{0}(z)+f_{1}(z)\right)
$$

with

$$
\begin{gathered}
f_{0}(z)=(z-1) \frac{t(z)}{z^{2}-t(z)}, f_{1}(z)=(z-1) \frac{(1-\beta) z^{2}}{z^{2}-t(z)} \frac{1-A(\phi(z))}{1-\phi(z)} \\
t(z)=\beta z \phi(z)+(1-\beta) z \phi(z) A(\phi(z)) .
\end{gathered}
$$

Let us focus on the behaviour of $f_{0}^{\prime}(z)$ as the function $f_{1}^{\prime}(z)$ can be analysed in a similar way. A combination of the results for $f_{0}^{\prime}(z)$ and $f_{1}^{\prime}(z)$ leads to (7). Clearly

$$
f_{0}^{\prime}(z)=-\frac{(t(z)-z)^{2}}{\left(z^{2}-t(z)\right)^{2}}+z^{2} \frac{(z-1)^{2} \sum_{k=0}^{\infty}(k+1) T_{k+2} z^{k}}{\left(z^{2}-t(z)\right)^{2}}
$$

with $T_{k}=\sum_{j \geqslant k} t_{j}$. Since the first term of $f_{0}^{\prime}(z)$ does not diverge for $z \rightarrow 1-$, we only need to take the second term into account. First we determine the asymptotic behaviour of $T_{k}$ by applying Theorem 1 to $t^{\prime \prime}(z)$. Observe that

$$
t^{\prime \prime}(z) \sim(1-\beta) z \phi(z) \frac{d^{2}}{d z^{2}}[A(\phi(z))] \text { for } z \rightarrow 1-.
$$

Let $\sigma=3-s$. We have

$$
\begin{aligned}
& \lim _{z \rightarrow 1-}(1-z)^{\sigma} \frac{d^{2}}{d z^{2}}[A(\phi(z))] \\
& =\lim _{z \rightarrow 1-}\left(\frac{1-z}{1-\phi(z)}\right)^{\sigma}(1-\phi(z))^{\sigma} A^{\prime \prime}(\phi(z))\left(\phi^{\prime}(z)\right)^{2} \\
& =\lim _{y \rightarrow 1-}(1-y)^{\sigma} A^{\prime \prime}(y)\left(\phi^{\prime}(1)\right)^{2-\sigma} .
\end{aligned}
$$

Applying Theorem [1 twice,

$$
A^{\prime \prime}(y) \sim \frac{1}{(1-y)^{\sigma}} a \Gamma(\sigma)
$$




$$
\sum_{k=0}^{n} k(k-1) t_{k} \sim \frac{a \Gamma(\sigma)}{\Gamma(\sigma+1)} n^{\sigma}\left(\phi^{\prime}(1)\right)^{2-\sigma}(1-\beta)
$$

we obtain

$$
T_{k} \sim{\frac{a^{\prime}}{\phi}}^{\prime}(1)^{s-1}(1-\beta) k^{1-s} .
$$

One more application of Theorem 1 results in

$$
f_{0}^{\prime}(z) \sim \frac{1}{\left(2-t^{\prime}(1)\right)^{2}} \frac{a \Gamma(3-s)}{(s-1) \Gamma(4-s)}(1-z)^{s-3} .
$$

Furthermore observe that

$$
(1-\rho)=\frac{2-t^{\prime}(1)}{(1-\beta) A^{\prime}(1)+1}
$$

hence

$$
f_{0}^{\prime}(z) \sim \frac{1}{(1-\rho)^{2}\left((1-\beta) A^{\prime}(1)+1\right)^{2}} \frac{a \Gamma(3-s)}{(s-1) \Gamma(4-s)}(1-z)^{s-3} .
$$

Using the Tauberian theorem for power series, the asymptotic behaviour of the coefficients of $Q^{\prime}(z)=\sum_{k} k q_{k} z^{k-1}$ follows from (7). Applying this theorem to $Q^{\prime}(z)$ results in

$$
q_{1}+2 q_{2}+\ldots n q_{n} \sim \frac{L}{\Gamma(4-s)} n^{3-s}
$$

Using [12 3.3 (c), pg. 59], we obtain

$$
\sum_{j>k} q_{j} \sim \frac{L}{\Gamma(4-s)} \frac{3-s}{s-2} k^{2-s}
$$

or

$$
\mathbf{P}\{q>k\} \sim \frac{a}{(s-1)(s-2)} \frac{1}{\mathbf{E}\left[\tau_{A}\right]+\mathbf{E}\left[\tau_{B}\right]}\left(\frac{\phi^{\prime}(1)^{s-1}}{1-\rho}+\phi^{\prime}(1)^{s-2}\right) k^{2-s} .
$$

Numerical example.

To illustrate how fast the asymptotic regime is reached we simulate the D-BMAP/D/1 queue with LRD arrival process characterized by $\mathbf{P}\left\{\tau_{A}=j\right\}=$ $(j+1)^{-s}-j^{-s}$, and $\phi(z)=1-\nu+\nu z$. Here $a=s-1$. For the first example $s=2.8, \beta=0.6$ and $\nu=0.3$, and for the second example $s=2.3, \beta=0.8$ and $\nu=0.3$. Hence the load of the system is about 0.68 for the first case and about 0.65 for the second case. As can be seen from Figure 1, the asymptotic regime is reached very quickly. The asymptotic behaviour of $\mathbf{P}\{q=k\}$ is given by

$$
\mathbf{P}\{q=k\} \sim \frac{a}{(s-1)} \frac{1}{\mathbf{E}\left[\tau_{A}\right]+\mathbf{E}\left[\tau_{B}\right]}\left(\frac{\phi^{\prime}(1)^{s-1}}{1-\rho}+\phi^{\prime}(1)^{s-2}\right) k^{1-s} .
$$




\subsection{The Tail Probabilities When $c>1$}

Contrary to the case $c=1$, no closed form formula describing the asymptotic behaviour can be obtained when $c>1$. The main reason is that an explicit formula for $Q(z)$ like (11) does not exist for the D-BMAP/D/c with $c>1$. Nevertheless it is possible to determine precisely the behaviour of the tail probabilities. It turns out that this behaviour depends on $c$ and on the sizes of the LRD and the SRD shares of the traffic mix.

First we derive an expression for the generating function of the stationary buffer distribution $q$, denoted by $Q(z)$, from the Pollachek-Kinchin equation for the $\mathrm{D}-\mathrm{BMAP} / \mathrm{D} / c$ queue.

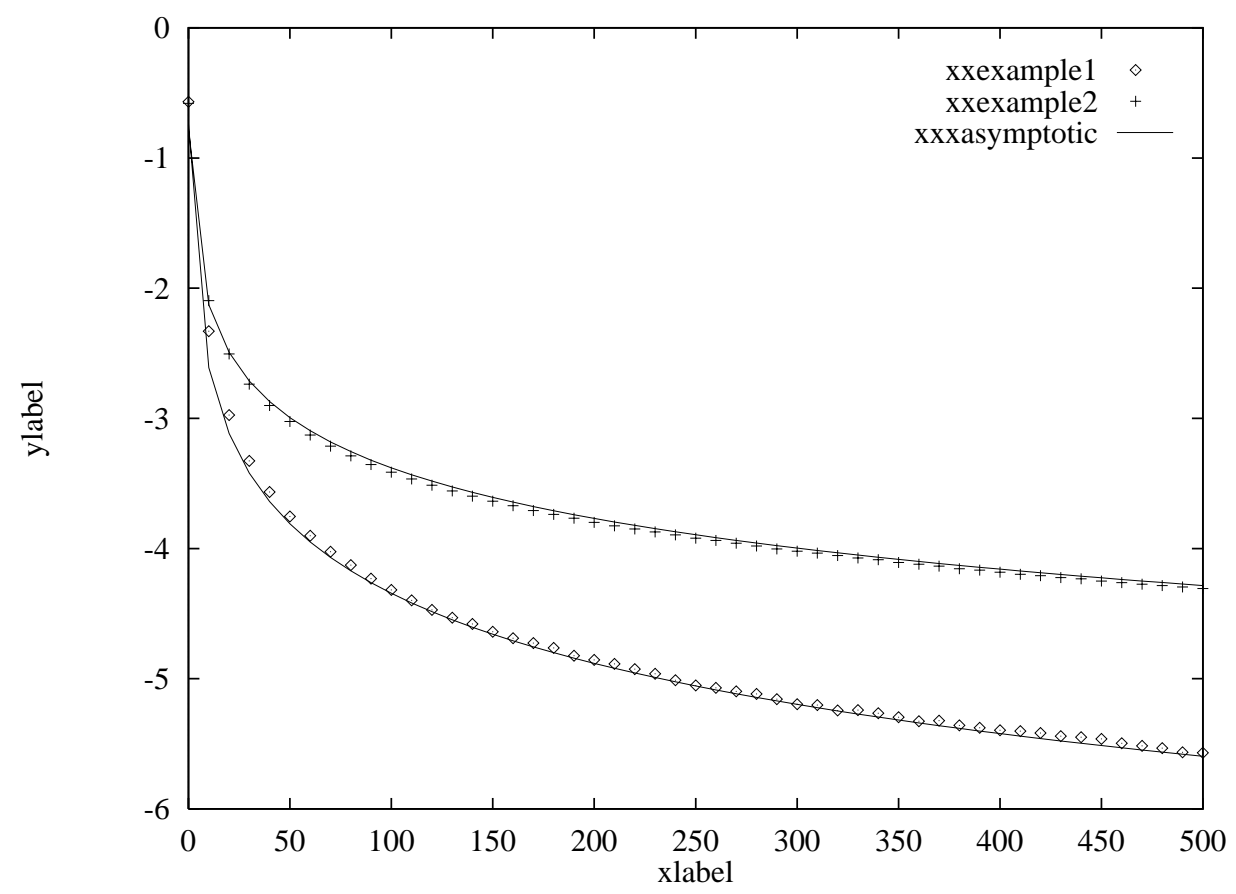

Fig. 1. Numerical example with $c=1$

Calculating $Q(z)$ When $c>1$. Recall that the Pollachek-Kinchin equation for the $\mathrm{D}-\mathrm{BMAP} / \mathrm{D} / c$ queue is given by

$$
\mathbf{X}(z)=\sum_{l=0}^{c-1} \mathbf{x}_{l} \mathbf{D}(z)\left(z^{c}-z^{l}\right)\left(z^{c} \mathbf{I}-\mathbf{D}(z)\right)^{-1} .
$$

Whereas for $c=1$ the vector $\mathbf{x}_{0}$ is known explicitly, here the vectors $\mathbf{x}_{0}, \ldots, \mathbf{x}_{c-1}$ can only be computed numerically, as is shown in [13, pg. 310-329]. This pre- 
vents us from deriving a closed form formula for $Q(z)=\mathbf{X}(z)$ e. Define for $l=0,1, \ldots, c-1$,

$$
\mathbf{w}^{(l)}(z)=\mathbf{x}_{l} \mathbf{D}(z)\left(z^{2} \mathbf{I}-\mathbf{D}(z)\right)^{-1},
$$

with $\mathbf{w}^{(l)}(z)=\left(w_{0}^{(l)} w_{1}^{(l)} \ldots\right)$. After some algebraic manipulations (see [3] for details) we obtain

$$
\begin{aligned}
\mathbf{w}^{(l)}(z) \mathbf{e}= & w_{0}^{(l)}(z)+w_{1}^{(l)}(z)\left[1+\sum_{k=1}^{\infty}\left(\sum_{j>k} a_{j}\right)\left(\frac{\phi(z)}{z^{c-1}}\right)^{k}\right] \\
& +\sum_{j=1}^{\infty}\left(\mathbf{x}_{l}\right)_{j} \sum_{k=j}^{\infty} \frac{1-\sum_{i=1}^{k} a_{i}}{1-\sum_{i=1}^{j-1} a_{i}}\left(\frac{\phi(z)}{z^{c-1}}\right)^{k-j+1} .
\end{aligned}
$$

The analysis of

$$
Q(z)=\sum_{l=0}^{c-1}\left(z^{c}-z^{l}\right) \mathbf{w}^{(l)}(z) \mathbf{e}
$$

reveals two different types of asymptotic behaviour. If $\phi^{\prime}(1)>c-1$, or equivalently, if the mean arrival rate of the background traffic exceeds $c-1$, then the tail probabilities decay according to a power-law, as is shown under Case 1. If the mean arrival rate of the background traffic is strictly less than $c-1$, then the tail probabilities decay approximately exponentially, as is demonstrated under Case 2. It turns out that the rate of this decay can be determined easily. For the case $\phi^{\prime}(1)=c-1$, the transition point, no conclusions can be drawn.

Case 1: $\phi^{\prime}(1)>c-1$. Since $\phi^{\prime}(1)>c-1$ we have

$$
\left.\frac{d}{d z}\left(\frac{\phi(z)}{z^{c-1}}\right)\right|_{z=1}>0
$$

Hence there exists an open set $G \subset D(0,1)$, with $D(0,1)$ the closed complex unit disk, having the following properties:

$$
\text { for each } z \in G:\left|\frac{\phi(z)}{z^{c-1}}\right|<1 \text {; }
$$

the interval $[\zeta, 1)$ belongs to $G$ for some $\zeta \in(0,1)$.

Hence on $G$ the representation (13) of $Q(z)$ can be used. Since we focus on the influence of the LRD on-off source, $\phi(z)$ is taken to be analytical on some open set containing $D(0,1)$.

We obtain the asymptotic behaviour of the tail probabilities, as in 3.1, by examining the behaviour of $Q^{\prime}(z)$. A detailed description of this analysis can be found in 3]. It is shown that for some $C>0$,

$$
Q^{\prime}(z) \sim \frac{C}{(1-z)^{3-s}} \text { for } z \rightarrow 1-,
$$


which implies

$$
\mathbf{P}\{q>k\} \sim \frac{C(3-s)}{(s-2) \Gamma(4-s)} k^{2-s} .
$$

The constant $C$ depends on $A(z), \beta, \phi(z), c$ and the vectors $\mathbf{x}_{0}, \ldots \mathbf{x}_{c-1}$. Keep in mind that $\mathbf{x}_{0}, \ldots, \mathbf{x}_{c-1}$ need a numerically calculation.

Case 2: $\phi^{\prime}(\mathbf{1})<c-1$. Contrary to the case $\phi^{\prime}(1)>c-1$, there exists some $z_{0}>1$ such that $\phi\left(z_{0}\right)=z_{0}$. We suppose that $z_{0}<\infty$, as $z_{0}=\infty$ would imply that the background traffic generates at most $c-1$ arrivals in a slot. If this is the case no buffer is needed, because all arriving cells can be served instantaneously. For $z \in\left(1, z_{0}\right)$,

$$
\frac{\phi(z)}{z^{c-1}}<1
$$

Since $\phi(z)$ is a generating function, it follows that $|\phi(z)|<\left|z^{c-1}\right|$, for all complex number $z$, with $1<|z|<\left|z_{0}\right|$. Furthermore, because $\phi(z) \neq z^{n}$ for each $n \geqslant 0$, there exist at most a finite number of complex points $z_{i}^{\star}$ with modulus 1 such that $\left|\phi\left(z_{i}^{\star}\right)\right|=\left|z_{i}^{\star}\right|$. Let $G$ be the maximal open set such that for $z \in G$,

$$
\left|\frac{\phi(z)}{z^{c-1}}\right|<1
$$

Hence $z \in G$, for all $1<|z|<\left|z_{0}\right|$. Furthermore $G \cap D(0,1)$ is non-empty. Notice that on $G$ the function $Q(z)$, given by (13), is well-defined. Recall that $q$ denotes the random variable associated with the stationary buffer distribution. Because $G \cap D(0,1)$ is non-empty, $Q(z)$ is a representation of the $z$-transform $\mathbf{E}\left[z^{q}\right]$ on $G$. Hence the power series $\sum_{k=0}^{\infty} q_{k} z^{k}$, associated with $\mathbf{E}\left[z^{q}\right]$, is holomorphic on the open disk with centre 0 and radius $\left|z_{0}\right|$. The point $z_{0}$ represents a non-removable singularity. Since on $G$,

$$
\mathbf{E}\left[z^{q}\right]=\sum_{k=0}^{\infty} q_{k} z^{k}=Q(z),
$$

it is possible to determine the asymptotic behaviour of $q_{k}=\mathbf{P}\{q=k\}$ by examining the behaviour of $Q^{\prime}(z)$. Define for $y \in(0,1)$,

$$
f(y)=Q^{\prime}\left(z_{0} y\right) .
$$

Analysing the behaviour of $f(y)$ for $y \rightarrow 1-$, by using similar arguments as for the $\phi^{\prime}(1)>c-1$ case, we obtain

$$
q_{1}+2 z_{0} q_{2}+\ldots+n z_{0}^{n-1} q_{n} \sim C n^{3-s},
$$

with $C>0$. If the sequence $\left(k z_{0}^{k-1} q_{k}\right)$ decreases, this implies

$$
\mathbf{P}\{q=k\} \sim(3-s) C z_{0}^{-k} k^{1-s} .
$$

In [4] this type of asymptotic behaviour is conjectured for similar queueing systems. 


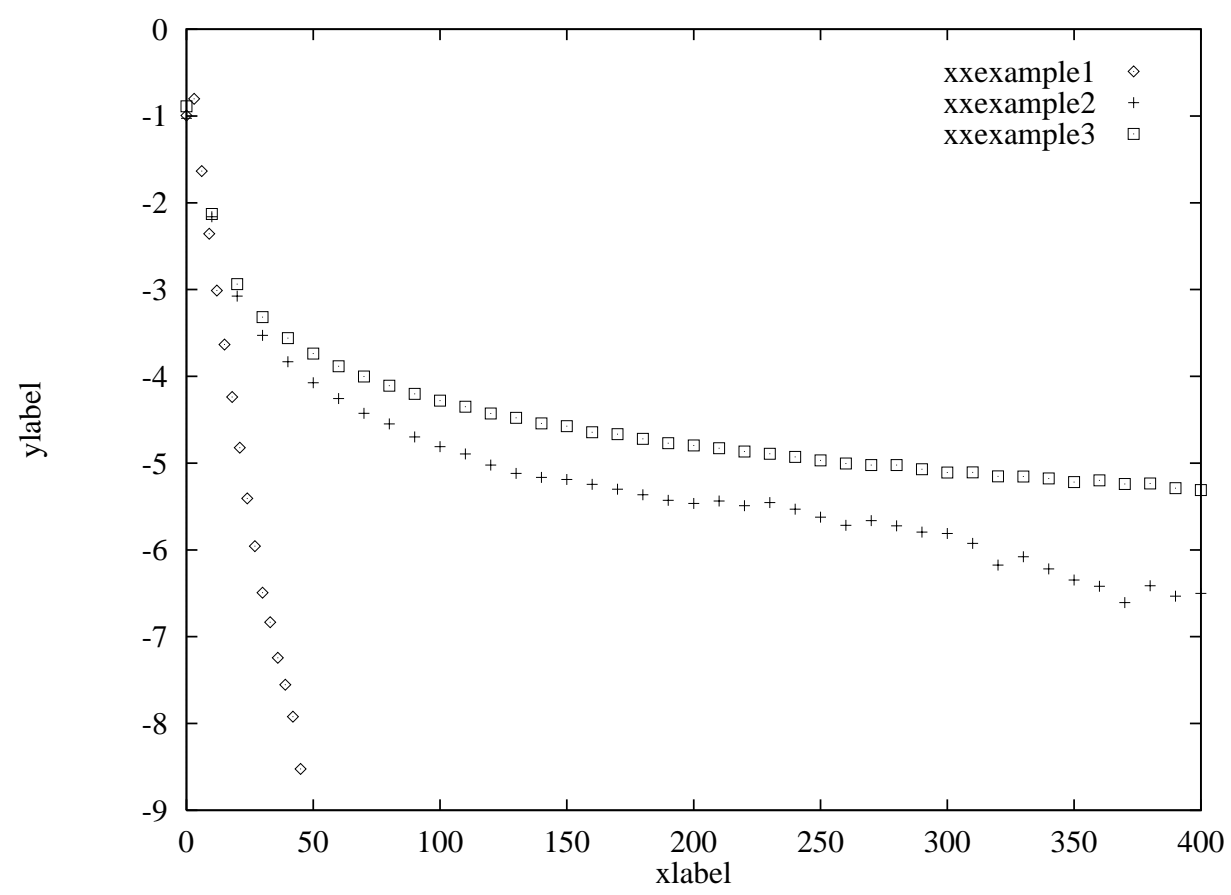

Fig. 2. Numerical example with $c=2$

Numerical Example. The tail probabilities for three examples, each one corresponding to a case considered above, are shown in Figure2. The on-periods are distributed as $\mathbf{P}\left\{\tau_{A}=j\right\}=(j+1)^{-s}-j^{-s}$, the background traffic is generated by Poisson variables with parameter $\lambda$. For the first example $s=2.3, \beta=0.4$ and $\lambda=0.8$, which results in a mean arrival rate of 1.5 . Clearly this example is covered by Case 2 . The second example has $s=2.6, \beta=0.4$ and $\lambda=1$. Hence the mean arrival rate is 1.6. Since $\lambda=1$, this example is a transition point case. In the third example $s=2.6, \beta=0.8$ and $\lambda=1.2$, hence mean arrival rate is 1.5. This leads to a power law decay.

\section{Conclusions}

In this paper we have investigated the decay of the tail of the buffer occupancy distribution for a c-server queue which is fed by a mix of long range and short range dependent traffic. It turns out that the decay may be exponentially or according to a power law, depending on the composition of the traffic mix and the number of parallel servers $c$. The transition point occurs when the $c>1$ and the arrival rate of the SRD traffic is exactly $c-1$. These results may be applied to traffic control schemes for LRD traffic in the following way. Consider SRD traffic mixed with LRD traffic which is spaced. The spacing distance is related 
to the value of the number of parallel servers $c$ in the model of the paper. The results give some insight under what conditions with respect to traffic mix and spacing distance, the tail of the buffer occupancy switches from a power law type to exponential.

\section{References}

1. C. Blondia : A discrete-time batch markovian arrival process as B-ISDN traffic model. Belgian Journal of Operations Research, Statistics and Computer Science. 32 (1993) 3-23

2. T. Daniëls and C. Blondia. Asymptotic behavior of a discrete-time queue with long range dependent input. In Proceedings of INFOCOM'99. (1999)

3. T. Daniëls and C. Blondia. Tail Transitions in Queues with Long Range Dependent Input. Technical Report, win-www.uia.ac.be/u/pats/publications.html. 2000

4. V. Dumas and A. Simonian. Asymptotic bounds for the fluid queue fed by subexponential on/off sources. Technical report, Mathématics Appliquées de Bordeaux. (1998)

5. A. Erramilli, O. Narayan, and W. Willinger. Experimental queueing analysis with long-range depdendent packet traffic. IEEE/ACM Trans. on Networking. 4 (1996) 209-223

6. W. Feller. An Introduction to Probability and Its Applications, Volume II. Wiley Series in Probability and Mathematical Statistics. John Wiley \& Sons, Inc. (1966)

7. H. J. Fowler and W. E. Leland. Local area network traffic characteristics, with implications for broadband network congestion management. IEEE JSAC 9 (1991) 1139-1149

8. M. W. Garret and W. Willinger. Analysis, modeling and generation of self-similar VBR video traffic. In Proceedings ACM Sigcomm'94 (1994) 269-280

9. D. Heath, S. Resnick, and G. Samorodnitsky. Heavy tails and long range dependence in on/off processes and associated fluid models. Technical report, Cornell University (1997)

10. P. R. Jelenković and A. A. Lazar. Asymptotic results for multiplexing on-off sources with subexponential on period. Advances in Applied Probability 31 (1999)

11. W. E. Leland, M. S. Taqqu, W. Willinger, and D. V. Wilson. On the self-similar nature of ethernet traffic (extended version). IEEE/ACM Trans. on Networking. 2 (1994) $1-15$

12. B. M. Makarov, M. G. Goluzina, A. A. Lodkin, and A. N. Podkorytov. Selected Problems in Real Analysis. Translations of Mathematical Monographs, American Mathematical Society 107 (1992)

13. M. F. Neuts. Structured stochastic matrices of the M/G/1-type and their applications. Marcel Dekker (1989)

14. J. Roberts, U. Mocci, and J. Virtamo. Broadband network teletraffic. Springer (1996)

15. M. S. Taqqu, W. Willinger, and R. Sherman. Proof of a fundamental result in self-similar traffic modeling. Computer Communication Review 27 (1997) 5-23 